\title{
Effects of Comorbidities, Inflammation and Glycaemic Control on Length of Hospital Stay/Potentially Avoidable Readmission Risk in Type 2 Diabetic Patients- A Retrospective Analysis
}

\author{
Irfan Karahan ${ }^{1}$ \\ ${ }^{1}$ Assistant Professor, Department of Internal Medicine, Kirikkale University, School of Medicine Kirikkale, Turkey.
}

\section{ABSTRACT}

\section{BACKGROUND}

Patients with diabetes can have long hospitalization and frequent readmissions for different reasons. Predicting the duration of hospitalization and readmission risk may be helpful for management. We wanted to identify factors that affect the length of hospital stay and potentially avoidable readmission risk.

\section{METHODS}

102 type 2 adults with diabetes that were hospitalized and discharged between July 2017 and July 2018 in Kırıkkale University, Department of Internal Medicine, were investigated retrospectively. HOSPITAL score was calculated for detecting 30-day readmission risk. Haemoglobin A1c for glycaemic control, C-reactive protein/serum albumin ratio (CAR) for inflammation, Charlson comorbidity score for multimorbidities, and age were researched as factors that affect the length of stay/ HOSPITAL score.

\section{RESULTS}

There were 70 female and 32 male patients. The median age of participants was 69 . Median HbA1c was 9.2\%, median Charlson comorbidity score was 6, median CAR was 3.33, median HOSPITAL score was 4. Significant positive correlation was detected between age, Charlson comorbidity score, CAR, HbA1c level and both hospital length of stay/HOSPITAL score by Spearman's correlation analysis. However, Charlson comorbidity score and CAR had significant values with both length of hospital stay/ HOSPITAL score according to the linear regression model.

\section{CONCLUSIONS}

Identifying the factors that affect readmission and length of stay, benefits hospitalized diabetes patient management. This can have favourable impact on costs, sources and approach. Type 2 diabetes patients can have multiple comorbidities and inflammatory conditions. Inflammation and comorbidity presence have negative effects on length of stay and avoidable readmission. Presence of these indices' in high levels should be considered high risk for readmission and these patients should be evaluated carefully.

\section{KEY WORDS}

Diabetes Mellitus, Hospitalization, Length of Stay, Admission, Hospital Score

\author{
Corresponding Author: \\ Irfan Karahan, \\ Assistant Professor \\ Department of Internal Medicine, \\ Kirikkale University of Medicine, \\ Yahsihan, Kirikkale-71450, Turkey. \\ E-mail:irfan_karahan@yahoo.com
}

DOI: $10.14260 /$ jemds $/ 2019 / 650$

Financial or Other Competing Interests: None.

How to Cite This Article:

Karahan I. Effects of comorbidities, inflammation and glycemic control on hospital length of stay/potentially avoidable readmission risk in type 2 diabetic patients- a retrospective analysis. J. Evolution Med. Dent. Sci. 2019;8(40):29912995, DOI: 10.14260/jemds/2019/650

Submission 06-08-2019,

Peer Review 17-09-2019,

Acceptance 25-09-2019,

Published 07-10-2019. 


\section{BACKGROUND}

Type 2 diabetes is a crucial healthcare problem with increasing prevalence. Frequencies of obesity and prediabetes have higher levels compared with past and continue to raise. ${ }^{1}$ Type 2 diabetes is a disease due to complex mechanisms and may require hospitalization for a variety of reasons. In addition to emergency situations such as acute conditions, hospitalization may be required in cases of chronic complications and triggering of cardiovascular diseases. ${ }^{2}$ Studies have shown that hospitalizations are much more common in patients with diabetes than in the normal population. The cost of this situation is also known for bringing a serious burden on health systems. ${ }^{3,4}$ Determining the factors affecting the length of hospital stay is important both for planning and taking measures. In a study to determine these factors, it was shown that heart failure, malignancies, advanced age, and comorbid diseases may have negative effects on hospital length of stay. ${ }^{5}$ It was noted that especially diabetic foot complications increased hospitalization and mortality. ${ }^{6}$ Hospital readmissions are a serious and frequent problem for healthcare systems. But some readmissions are avoidable and detecting patients at high risk of potential readmission may have benefits. ${ }^{7}$ Unfortunately, recent studies showed healthcare providers could not identify patients at high risk. Readmission is a complex and important problem for patients with diabetes. Age, ethnicity, comorbidities, environmental factors, etc; can affect hospitalization and readmissions. ${ }^{8}$ Detecting the factors affecting the hospital length of stay and potentially avoidable readmission is crucial for hospitalization management of patients with diabetes. In the present study, it was aimed to investigate the effects of inflammation markers, comorbidities presence, glycaemic control on the length of hospital stay, and potentially avoidable readmission risk.

\section{METHODS}

The present study was designed as retrospective analysis and included 102 adult patients with type 2 diabetes who were hospitalized and discharged from Kırıkkale University, School of Medicine, Internal Medicine Clinic between July 2017 and July 2018, which were retrospectively researched. Age of patients; gender; comorbid diseases; biochemistry parameters, HbA1c levels, C-reactive protein (CRP)/albumin ratio on the first day of admission were evaluated. Charlson comorbidity index was used for comorbidity assessment. Charlson comorbidity index also contains age, myocardial infarction history, congestive heart failure, peripheral vascular disease, cerebrovascular accident, dementia, peptic ulcer disease, liver disease, end-organ sequela of diabetes mellitus, hemiplegia, chronic kidney disease, solid tumour, leukemia, lymphoma, and AIDS parameters. The patients who have these parameters take more points and lower estimated 10-year survival. HOSPITAL score was calculated for detecting potentially avoidable readmission of the patients. HOSPITAL score includes haemoglobin level at discharge, discharge from an oncology service, sodium level at discharge, procedure during the index admission, index type of admission, number of admissions during the last 12 months, and length of hospital stay. CRP/ albumin ratio was used as an inflammation marker. The relationship between the length of stay, HOSPITAL score, and HbA1c, CRP/ albumin ratio, Charlson comorbidity score. The study was approved by the Institutional Review Board of Kırıkkale University, School of Medicine for ethical consideration.

Statistical Package for the Social Sciences version 25 was used for all statistical analysis. Shapiro-Wilk test was used to evaluate the distribution of descriptive data. Median and minimum-maximum values were calculated for data not normally distributed. Mann Whitney U test was performed for two-group comparison about not normally distributed variables. The relationship between numerical data was evaluated by Spearman's correlation analysis. Multiple linear regression analysis was performed with significant data of correlation for analyzing causality. $\mathrm{p}<0.05$ was considered as significant.

\section{RESULTS}

The median age was 69 of all 102 individuals. There were 70 female (68.6\%), and 32 male patients (31.4\%). Median HbA1c was detected as $\% 9.2$, median Charlson comorbidity score was calculated as 6, median CRP was detected as $13 \mathrm{mg} / \mathrm{L}$, median plasma albumin level was detected as $3.3 \mathrm{~g} / \mathrm{dl}$ (2.2-5), median $\mathrm{CRP} /$ albumin ratio was calculated as 3.33 , median HOSPITAL score was calculated as 4 (Table 1). Data showed that female and male patients had similar length of hospital stay [9 days (3-97) vs. 8.5 days (3-45), $\mathrm{p}=0.87$ ] and HOSPITAL score [4 (08) vs 3 (1-7), $p=0.79]$. The significant positive correlation was detected between age, Charlson comorbidity score, CAR, HbA1c level and hospital length of stay $(r=0.309, p=0.002 ; r=-$ $0.208, \quad \mathrm{p}=0.03 ; \mathrm{r}=0.593, \mathrm{p}<0.001 ; \quad \mathrm{r}=0.472, \mathrm{p}<0.001$ respectively) by Spearman's correlation analysis. The significant positive correlation was detected between age, Charlson comorbidity score, CRP/ albumin ratio, HbA1c level and HOSPITAL score $(\mathrm{r}=0.168, \mathrm{p}=0.09 ; \mathrm{r}=-0.198, \mathrm{p}=0.04$; $\mathrm{r}=0.438, \mathrm{p}<0.001 ; \mathrm{r}=0.564, \mathrm{p}<0.001$ respectively).

According to multiple linear regression analysis; age and HbA1c did not have statistically significant values, Charlson comorbidity score and CRP/ albumin ratio had significant values with both length of stay/HOSPITAL score (Table 2) (Figures 1, and 2).

\begin{tabular}{|c|c|}
\hline Age, year & $69(32-92)$ \\
\hline Female/ male (n) & $70 / 32$ \\
\hline Length of hospital stay, day & $9(3-97)$ \\
\hline HOSPITAL score & $4(0-8)$ \\
\hline Charlson comorbidity score & $6(9-1-11)$ \\
\hline HbA1c \% & $9.2(5.1-17.7)$ \\
\hline CRP/albumin ratio & $3.33(0.01-123.89)$ \\
\hline Table 1. Patient Characteristics and Laboratory on Admission \\
\hline
\end{tabular}

\begin{tabular}{|c|c|c|c|c|c|c|c|c|c|c|}
\hline & \multicolumn{7}{|c|}{ LOS } & \multicolumn{5}{c|}{ HOSPITAL Score } \\
\hline & B & SE & $\boldsymbol{\beta}$ & t & Sig. & B & SE & $\boldsymbol{\beta}$ & t & Sig. \\
\hline Age & -0.112 & 0.131 & -0.108 & -0.86 & $\mathrm{p}=0.39$ & -0.30 & 0.017 & -0.222 & -1.73 & $\mathrm{p}=0.08$ \\
\hline HbA1c & -0.435 & 0.478 & -0.085 & -0.91 & $\mathrm{p}=0.36$ & -0.82 & 0.064 & -0.122 & -1.29 & $\mathrm{p}=0.20$ \\
\hline $\begin{array}{c}\text { Charlson } \\
\text { comor- } \\
\text { bidity } \\
\text { score }\end{array}$ & 1.965 & 0.742 & 0.347 & 2.64 & $\mathrm{p}=0.009$ & 0.362 & 0.099 & 0.485 & 3.65 & $\mathrm{p}<0.001$ \\
\hline CAR & 0.169 & 0.048 & 0.327 & 3.49 & $\mathrm{p}=0.001$ & 0.013 & 0.006 & 0.196 & 2.06 & $\mathrm{p}=0.04$ \\
\hline \multicolumn{10}{|c|}{ Table 2. Multiple Linear Regression Model } \\
\hline $\begin{array}{l}\text { HbA1c: Haemoglobin A1c CAR: C-reactive protein/albumin ratio Sig: Significance } \\
\text { SE: Standart error }\end{array}$ \\
\hline
\end{tabular}



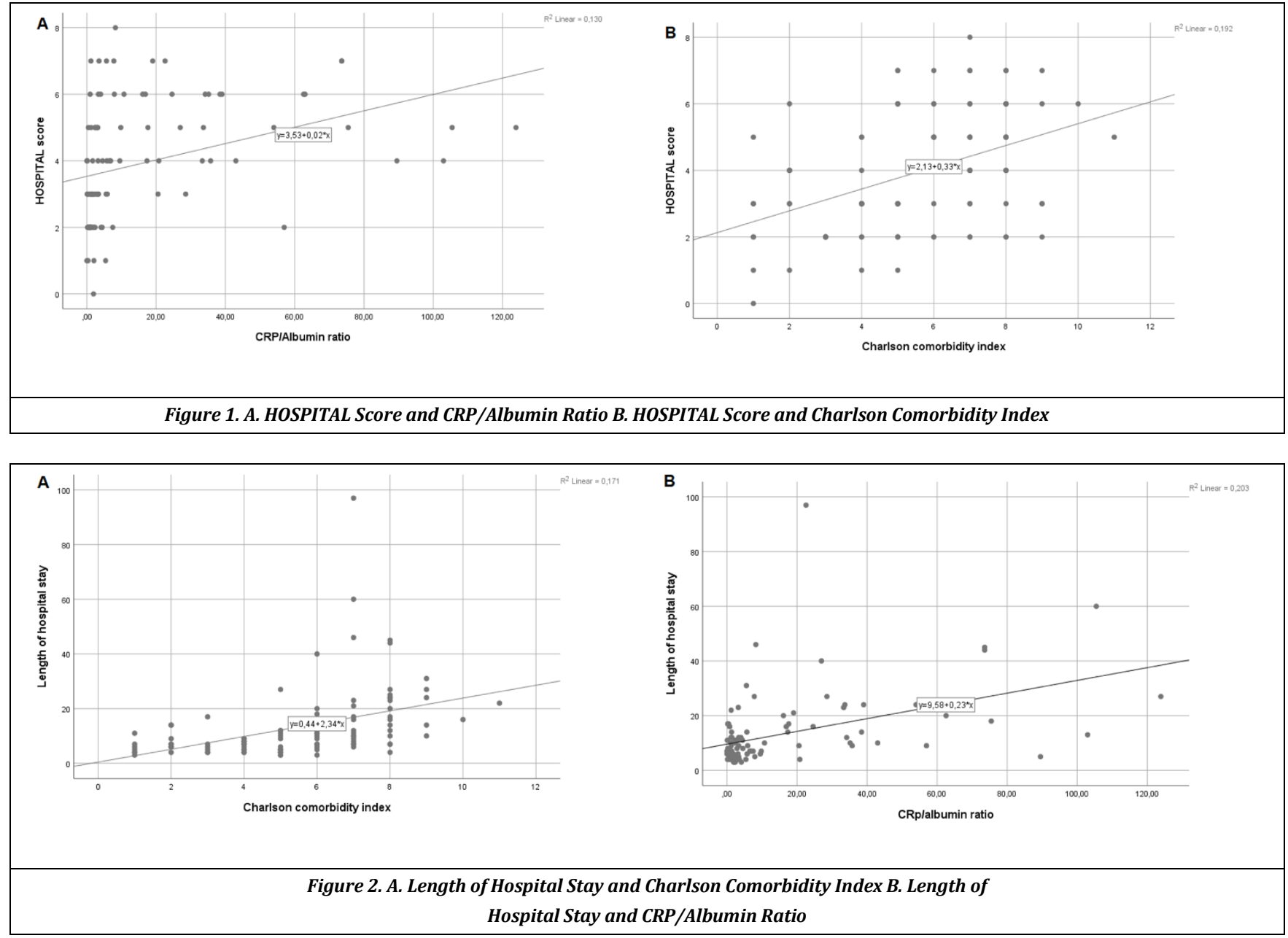

\section{DISCUSSION}

In this study, we showed that comorbidities and high inflammation markers' levels could increase the length of hospital stay and 30-day potentially avoidable readmission risk. Longer hospital staying may be associated with antibiotic usage, procedures due to hospital conditions and chronic complications. Patients with diabetes may have multiple comorbidities and they may affect HOSPITAL score components. Comorbidities, drugs, complications of diabetes can cause anaemia, electrolyte disorders, and acute admissions. Therefore, HOSPITAL score could have been calculated as higher levels. The regression model showed the main impacting factors were CRP/ albumin ratio and comorbidities, although age and $\mathrm{HbA} 1 \mathrm{c}$ have significant values due to correlation analysis. Also, it can be explained by raising age and HbA1c with inflammation and comorbidities without causality. The gender did not affect both duration and readmission risk. There are not many studies in the literature that directly investigate factors affecting hospital stay and readmission in patients with diabetes.

Cromarty et al. 9 showed that diabetes patients had consistently longer LOS and a higher rate of hospital-acquired diagnoses than similar patients with chronic conditions. Especially patients with end-organ sequela had a longer duration of hospitalization. Enomoto et al.10 detected that comorbidities like congestive heart failure, rheumatoid diseases, peripheral vascular disease, renal diseases, cancer may affect length of stay and 30-day readmission. They support our findings. Primarily frequent readmission diagnoses were infectious diseases, cardiac and respiratory diseases in that mentioned study. Gillani et al. ${ }^{11}$ researched non-elective readmissions to hospital in people with diabetes as similar to our findings. The majority of re-admissions causes were due to multimorbidity. But re-admission causes were often related to non-diabetic conditions. They suggested readmissions were justified and avoidable. We found $\mathrm{HbA1c}$ levels were not effective on readmission. Oppose to our data, some studies showed that tight glycaemic control and escalation of diabetes treatment decreased readmission rates.12,13 This result can be related to our population characteristics and disease presentations. Inversely, another study showed that decrease haemoglobin A1c was related to higher 30-day readmission rates in acute heart failure patients. ${ }^{14}$ Other factors were found as effective factors on readmission. Jiang et al. ${ }^{15}$ showed that racial/ethnic disparities are more evident in 180-day than in 30-day readmission rates, readmission diagnoses vary by race/ethnicity, with Blacks and Hispanics at higher risk for those complications. Another study showed that inpatient diabetes education was independently related to a lower frequency of hospital readmission within 30 days; this relationship was attenuated by 180 days. ${ }^{16}$

Dashtpour et al. ${ }^{17}$ conducted a study fort o investigate individual factors which related to readmissions in Iran. They found that aging, number of children, occupational status, exercise, body mass index, smoking were influencers about readmission of the patients with type 2 diabetes. 
Diabetes Early Readmission Risk Indicator (DERRI ${ }^{\mathrm{TM}}$ ) was also developed for predicting the risk of all-cause readmission within 30 days among hospitalized patients with diabetes. By this tool, ten predictors were identified: living within 5 miles of the hospital; employment status; insulin use before admission; macrovascular complications of diabetes; serum haematocrit, creatinine, and sodium on admission; history of hospital discharge within 90 days before admission; most recent discharge up to one year before admission; and anaemia. 18,19 In the present study HOSPITAL score was utilized for detecting 30-day readmission risk. The "HOSPITAL" score described patients at high risk of 30-day potentially avoidable readmission with moderately high discrimination and good calibration when applied to a large international multicenter cohort of medical patients. ${ }^{20,21}$ Further studies will be able to show sensitivity and predictivity differences of these tools.

Our study may be first research for the effects of specific factors on both hospital length of stay and potentially avoidable readmission. But multi-center, more extensive and prospective trials should be needed for detecting other factors.

This study has several limitations. It is a retrospective analysis and single-center experience. Medical records, patient characteristics, full laboratory values, and history of disease were not completely available. There was a lack of knowledge about the microvascular and macrovascular complications of diabetes. The results did not reflect readmission, just reflected HOSPITAL score results and "risk". Although international validation was made, HOSPITAL score was not validated for Turkish people and this was an important limitation for this study. Further investigations are needed to clarify the evaluation of other factors which affect the duration of hospitalization and readmission risk.

\section{CONCLUSIONS}

Defining the factors that affect the length of stay and avoidable readmission, may be crucial for planning qualified intervention. Patients with type 2 diabetes can have multiple comorbidities and inflammatory conditions than the population without diabetes. Inflammation and comorbidity presence have negative effects on the length of stay and avoidable readmission. Patients who have these conditions should be considered as a higher risk group and healthcare resources should be used in a more planned manner.

\section{REFERENCES}

[1] Satman I, Omer B, Tutuncu Y, et al. Twelve-year trends in the prevalence and risk factors of diabetes and prediabetes in Turkish adults. Eur J Epidemiol 2013;28(2):169-80.

[2] Khalid JM, Raluy-Callado M, Curtis BH, et al. Rates and risk of hospitalisation among patients with type 2 diabetes: Retrospective cohort study using the UK General Practice Research Database linked to English Hospital Episode Statistics. Int J Clin Pract 2014;68(1):40-8.
[3] Aro S, Kangas T, Reunanen A, et al. Hospital use among diabetic patients and the general population. Diabetes Care 1994;17(11):1320-9.

[4] Carral F, Olveira G, Salas J, et al. Care resource utilization and direct costs incurred by people with diabetes in a Spanish hospital. Diabetes Res Clin Pract 2002;56(1):2734.

[5] Lara-Rojas CM, Pérez-Belmonte LM, López-Carmona MD, et al. National trends in diabetes mellitus hospitalization in Spain 1997-2010: analysis of over 5.4 millions of admissions. Eur J Intern Med 2019;60:83-9.

[6] Nirantharakumar K, Saeed M, Wilson I, et al. In-hospital mortality and length of stay in patients with diabetes having foot disease. J Diabetes Complications 2013;27(5):454-8.

[7] Aubert CE, Schnipper JL, Williams MV, et al. Simplification of the HOSPITAL score for predicting 30-day readmissions. BMJ Qual Saf 2017;26(10):799-805.

[8] Harris MI, Eastman RC, Cowie CC, et al. Racial and ethnic differences in glycemic control of adults with type 2 diabetes. Diabetes Care 1999;22(3):403-8.

[9] Cromarty J, Parikh S, Lim WK, et al. Effects of hospitalacquired conditions on length of stay for patients with diabetes. Intern Med J 2014;44(11):1109-16.

[10] Enomoto LM, Shrestha DP, Rosenthal MB, et al. Risk factors associated with 30-day readmission and length of stay in patients with type 2 diabetes. J Diabetes Complications 2017;31(1):122-7.

[11] Gillani SMR, Aziz U, Blundell D, et al. Non elective readmissions to an acute hospital in people with diabetes: Causes and the potential for avoidance. The WICKED project. Prim Care Diabetes 2015;9(5):392-6.

[12] Boreland L, Scott-Hudson M, Hetherington K, et al. The effectiveness of tight glycemic control on decreasing surgical site infections and readmission rates in adult patients with diabetes undergoing cardiac surgery: a systematic review. Hear Lung 2015;44(5):430-40.

[13] Eby E, Hardwick C, Yu M, et al. Predictors of 30 day hospital readmission in patients with type 2 diabetes: a retrospective, case-control, database study. Curr Med Res Opin 2015;31(1):107-14.

[14] Núñez J, Bonanad C, Navarro JP, et al. Differential effect of glycosylated haemoglobin value and antidiabetic treatment on the risk of 30-day readmission following a hospitalization for acute heart failure. Rev Española Cardiol (English Ed) 2015;68(10):852-60.

[15] Jiang HJ, Andrews R, Stryer D, et al. Racial/ethnic disparities in potentially preventable readmissions: the case of diabetes. Am J Public Health 2005;95(9):1561-7.

[16] Healy SJ, Black D, Harris C, et al. Inpatient diabetes education is associated with less frequent hospital readmission among patients with poor glycemic control. Diabetes Care 2013;36(10):2960-7.

[17] Dashtpour V, Hesaraki M, Abavisani M, et al. Investigating individual factors related to readmission of patients with type 2 diabetes - a cross-sectional study. J Evol Med Dent Sci 2018;7(53):5604-9.

[18] Rubin DJ, Handorf EA, Golden SH, et al. Development and validation of a novel tool to predict hospital readmission risk among patients with diabetes. Endocr Pract 2016;22(10):1204-15. 
[19] Rubin DJ, Donnell-Jackson K, Jhingan R, et al. Early readmission among patients with diabetes: a qualitative assessment of contributing factors. J Diabetes Complications 2014;28(6):869-73.

[20] Donze JD, Williams MV, Robinson EJ, et al. International validity of the "HOSPITAL" score to predict 30-day potentially avoidable readmissions in medical patients. J Am Med Assoc Intern Med 2016;176(4):496-502.
[21] Donze J, Aujesky D, Williams D, et al. Potentially avoidable 30-day hospital readmissions in medical patients: derivation and validation of a prediction model. JAMA Intern Med 2013;173(8):632-8. 